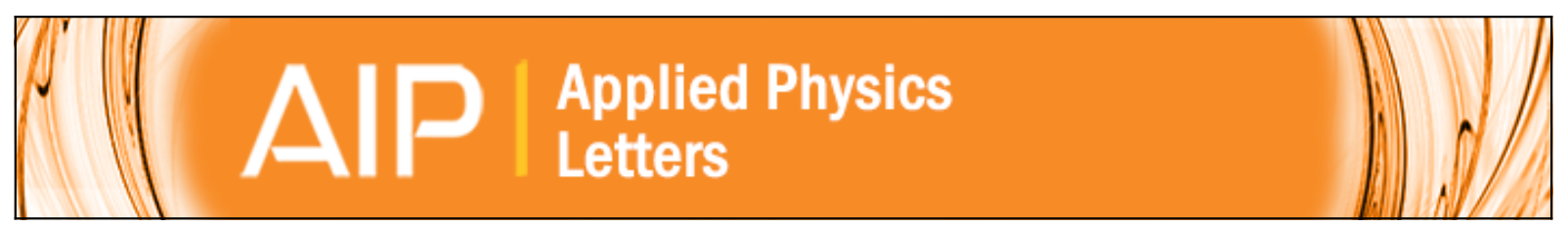

\title{
Enhanced bipolar fatigue resistance in CaZrO3-modified (K,Na)NbO3 lead-free piezoceramics
}

Fang-Zhou Yao, Eric A. Patterson, Ke Wang, Wook Jo, Jürgen Rödel, and Jing-Feng Li

Citation: Applied Physics Letters 104, 242912 (2014); doi: 10.1063/1.4884826

View online: http://dx.doi.org/10.1063/1.4884826

View Table of Contents: http://scitation.aip.org/content/aip/journal/apl/104/24?ver=pdfcov

Published by the AIP Publishing

\section{Articles you may be interested in}

Effect of poling temperature on piezoelectricity of $\mathrm{CaZrO3-modified}(\mathrm{K}, \mathrm{Na}) \mathrm{NbO}$-based lead-free ceramics J. Appl. Phys. 116, 114102 (2014); 10.1063/1.4895774

Hot-stage transmission electron microscopy study of $(\mathrm{Na}, \mathrm{K}) \mathrm{NbO} 3$ based lead-free piezoceramics Appl. Phys. Lett. 105, 042904 (2014); 10.1063/1.4891960

Fatigue-free unipolar strain behavior in $\mathrm{CaZrO3}$ and $\mathrm{MnO} 2$ co-modified $(\mathrm{K}, \mathrm{Na}) \mathrm{NbO}$-based lead-free piezoceramics

Appl. Phys. Lett. 103, 192907 (2013); 10.1063/1.4829150

Phase transitions, relaxor behavior, and large strain response in LiNbO3-modified Bi0.5(Na0.80K0.20)0.5TiO3 lead-free piezoceramics

J. Appl. Phys. 114, 044103 (2013); 10.1063/1.4816047

Comprehensive investigation of elastic and electrical properties of $\mathrm{Li} / \mathrm{Ta}$-modified $(\mathrm{K}, \mathrm{Na}) \mathrm{NbO} 3$ lead-free piezoceramics

J. Appl. Phys. 113, 174105 (2013); 10.1063/1.4803711

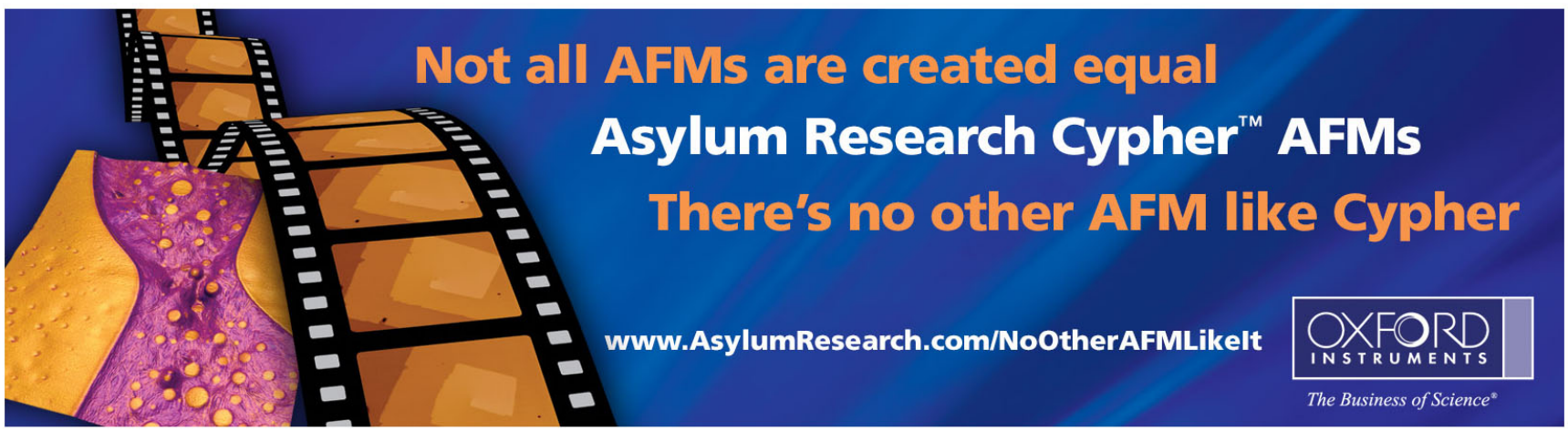




\title{
Enhanced bipolar fatigue resistance in $\mathrm{CaZrO}_{3}$-modified $(\mathrm{K}, \mathrm{Na}) \mathrm{NbO}_{3}$ lead-free piezoceramics
}

\author{
Fang-Zhou Yao, ${ }^{1}$ Eric A. Patterson, ${ }^{2}$ Ke Wang, ${ }^{1, a)}$ Wook Jo, ${ }^{2}$ Jürgen Rödel, ${ }^{2}$ \\ and Jing-Feng $\mathrm{Li}^{1, \mathrm{~b})}$ \\ ${ }^{1}$ State Key Laboratory of New Ceramics and Fine Processing, School of Materials Science and Engineering, \\ Tsinghua University, Beijing 100084, China \\ ${ }^{2}$ Institute of Materials Science, Technische Universität Darmstadt, Darmstadt 64287, Germany
}

(Received 17 April 2014; accepted 10 June 2014; published online 20 June 2014)

\begin{abstract}
The bipolar fatigue behavior of $(\mathrm{K}, \mathrm{Na}) \mathrm{NbO}_{3}(\mathrm{KNN})$-based lead-free piezoceramics was investigated. A comparative analysis demonstrated that $\mathrm{CaZrO}_{3}$-modified $\mathrm{KNN}$ ceramics exhibited highly enhanced bipolar fatigue resistance due to the reduced lattice distortion (c/a ratio) and coexistence of orthorhombic and tetragonal phases. The hypothesis was verified by systematical studies of cycle-dependent large and small signal parameters and micro-morphologies. It was identified that mechanical stress induced microstructure damage beneath the electrodes renders the KNN ceramics to be vulnerable to bipolar cycling; while the mild fatigue behavior for $\mathrm{CaZrO}_{3}$-modified ones mainly originates from a thermally reversible domain wall pinning. (C) 2014 AIP Publishing LLC.

[http://dx.doi.org/10.1063/1.4884826]
\end{abstract}

The milestone marked by Saito et al. ${ }^{1}$ in $(\mathrm{K}, \mathrm{Na}) \mathrm{NbO}_{3}$ (KNN)-based lead-free piezoceramics has won the system considerable opportunities to be one of the most promising candidates to substitute the currently dominating but environmentally hazardous $\mathrm{Pb}(\mathrm{Zr}, \mathrm{Ti}) \mathrm{O}_{3}$ (PZT) family. Consequently, it has triggered worldwide interest, ${ }^{1-4}$ through which significant resources were devoted to strengthening their piezoelectricity through compositional optimization or domain engineering. ${ }^{5-9}$ However, the essential requirements enabling piezoceramics to be appropriate for applications comprise not only reproducibly outstanding piezoelectric properties but also long term stability of these properties, e.g., good fatigue resistance. ${ }^{10}$ Bipolar piezoelectric fatigue is manifested by the loss of switchable polarization and the decrease of strain and piezoelectric coefficient of piezoceramics after being exposed to millions of electric field cycles. It has been extensively studied in $\mathrm{PZT}^{10-12}$ and $\left(\mathrm{Bi}_{0.5} \mathrm{Na}_{0.5}\right) \mathrm{TiO}_{3}$ (BNT)based $^{13-15}$ ceramics, whereas less fatigue data are available for the KNN system. ${ }^{16,17}$ To elucidate the fatigue behavior of piezoceramics, several mechanisms were proposed in the literature, which included domain wall pinning by point defects, ${ }^{18,19}$ field screening resulting from the formation of a dead layer underneath the electrodes ${ }^{20}$ or microstructure damage in the material adjacent to the electrodes as a result of large mechanical stresses during the domain switching. ${ }^{21}$

As fatigue-free behavior for piezoceramics is highly desirable, two possible strategies have been adopted so far to improve the fatigue resistance in two separate systems. In the first case, piezoceramics were fabricated to avoid the morphotropic phase boundary (MPB) or polymorphic phase transition (PPT) at the cost of sacrificing piezoelectric performance. This has been shown both in PZT with single phase rhombohedral symmetry, which has small internal stress associated with the shear distortion of the cubic lattice $^{22}$ and in KNN for single phase orthorhombic symmetry. ${ }^{23}$

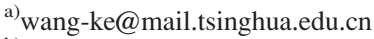

b)jingfeng@mail.tsinghua.edu.cn
}

Alternatively, efforts have been made to reduce the concentration of intrinsic defects to prevent domain pinning due to agglomeration from repeated cycling. ${ }^{14}$ It is well recognized that either the $\mathrm{PPT}^{23,24}$ or $\mathrm{MPB}^{7,25}$ effect is indispensable for enhancing piezoelectricity in KNN-based ceramics. Thus, at first glance it seems to be contradictory to deliver both promising piezoelectric performance and high fatigue resistance in the KNN system via the first method.

In this work, a feasible solution was validated for improving the bipolar fatigue resistance of KNN-based piezoceramics near the polymorphic phase boundary, namely, by reducing the lattice distortion through $\mathrm{CaZrO}_{3}$ modification. Two compositions of $(100-x)(\mathrm{K}, \mathrm{Na}, \mathrm{Li})(\mathrm{Ta}, \mathrm{Nb}) \mathrm{O}_{3}-\mathrm{xCaZrO}_{3}$ (with $\mathrm{x}=0$ and 5 , denoted by CZ0 and CZ5, respectively) were chosen as the objects of study. It was found that CZ5 ceramics possess much superior bipolar fatigue resistance to $\mathrm{CZ0}$, benefiting from insignificant mechanical stress induced by the lowered lattice distortion and increased mixture of orthorhombic and tetragonal phases.

Ceramic samples with the nominal composition of $(100-\mathrm{x})\left(\mathrm{K}_{0.49} \mathrm{Na}_{0.49} \mathrm{Li}_{0.02}\right)\left(\mathrm{Ta}_{0.2} \mathrm{Nb}_{0.8}\right) \mathrm{O}_{3}-\mathrm{xCaZrO}_{3}(\mathrm{CZx})$ with 2 wt. $\% \mathrm{MnO}_{2}$ addition were prepared by a conventional ceramic processing route as described elsewhere. ${ }^{26}$ The assintered bulk discs were ground to $1 \mathrm{~mm}$ in thickness and the surfaces of the pellets were polished with diamond paste down to $1 \mu \mathrm{m}$. High-resolution X-ray diffraction (XRD; Rigaku, D/Max250, Tokyo, Japan) measurements within the $2 \theta$ range from $20^{\circ}$ to $60^{\circ}$ were conducted with a scanning speed of $0.02^{\circ} / \mathrm{s}$ to determine the crystal structure by using $\mathrm{Cu}$ $K \alpha$ radiation $(\lambda=1.5406 \AA)$. To ensure good interface contact, Ag layers with a thickness of $50 \mathrm{~nm}$ were deposited onto both sides of the samples by sputtering. Silver paste was subsequently painted onto the deposited Ag layers and fired at $550^{\circ} \mathrm{C}$ for $30 \mathrm{~min}$ to protect the electrode from scratches. The samples were fatigued with a bipolar sinusoidal electric signal with the amplitude of $2 \mathrm{kV} / \mathrm{mm}$ (which corresponds to about two times the coercive field, $2 E_{\mathrm{C}}$ ) at $50 \mathrm{~Hz}$ up to $1 \times 10^{7}$ cycles. It should be mentioned that no prepoling was applied 
before the first measurement. Before increasing orders of cycling number, the fatigue process was stopped for measurement of large signal parameters polarization $P_{3}$ and strain $S_{33}$ as well as small signal parameters piezoelectric coefficient $d_{33}$ and permittivity $\varepsilon_{33}$ (tensor notations are taken). The large signal hysteresis for $P_{3}$ and $S_{33}$ were recorded using a bipolar triangular electrical signal with the maximum field amplitude of $4 \mathrm{kV} / \mathrm{mm}$ (approximately four times the coercive field, $\left.4 E_{\mathrm{C}}\right)$ at the frequency of $1 \mathrm{~Hz}$, while the small signal fielddependent $d_{33}$ and $\varepsilon_{33}$ were recorded with a triangular signal of $4 \mathrm{kV} / \mathrm{mm}$ and a frequency of $1 \mathrm{~Hz}$, over which an AC voltage of $25 \mathrm{~V}$ and $250 \mathrm{~Hz}$ was superimposed. The fatigue, large signal hysteresis, and small signal hysteresis measurements were done using a commercial ferroelectric analyzer test setup (TF1000, aixACCT Systems GmbH, Germany). The microstructures of fatigued samples were investigated by means of scanning electron microscopy (SEM, JEOL JSM7001,Tokyo, Japan). To clarify the effect of annealing treatment on fatigue behavior, the samples fatigued to $10^{7}$ cycles were annealed at $500^{\circ} \mathrm{C}$ for $4 \mathrm{~h}$, and then the measurements were performed again.

Figure 1 shows the XRD patterns of CZ0 and CZ5 ceramics. Generally, the proportions of the phase structures present in KNN-based piezoceramics can be roughly estimated by assessing the relative intensities of $\langle 200\rangle$ peaks $\left(I_{002} / I_{200}\right)$ around $2 \theta=45^{\circ} .^{3,27}$ For randomly oriented piezoceramics with an orthorhombic symmetry, the ideal ratio of $I_{002} / I_{200}$ equals to $2: 1$, while it evolves to $1: 2$ for a tetragonal phase. ${ }^{27}$ The profiles of the $\langle 200\rangle$ peaks reveal that CZ0 and CZ5 ceramics have distinct structures. The coexistence of orthorhombic and tetragonal phases was indicated by the peak splitting of both CZ0 and CZ5 ceramics, but an orthorhombic symmetry is dominant in CZ0. The enlarged separation of the (200) peak from the (002) in Fig. 1(b) suggests that the crystal structure of the CZ0 system had a higher c/a ratio, while it was closer to a pseudocubic structure for CZ5 ceramics, as schematically represented in the insets of Fig. 1(a). Combined with the similar intensities of the signals of the $\langle 200\rangle$ peaks for CZ5, this pseudocubic structure is proposed to be composed of nearly equal parts of orthorhombic and tetragonal phases. The beta angle in orthorhombic KNN

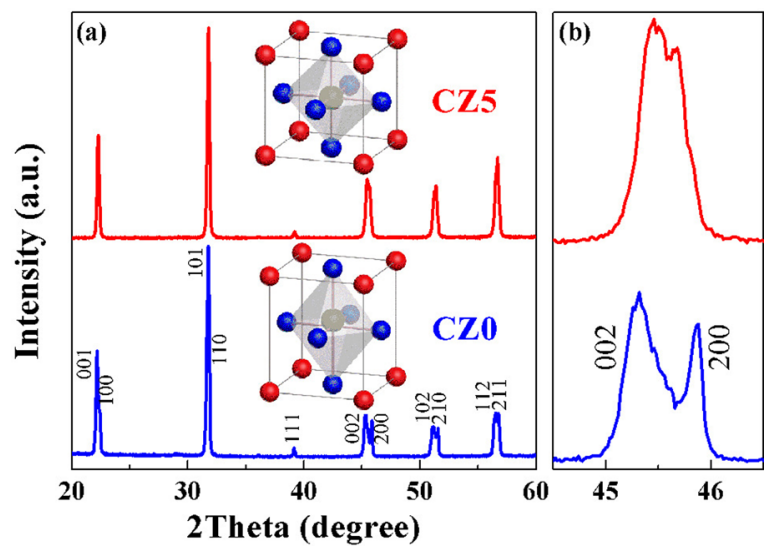

FIG. 1. (a) XRD patterns of CZ0 and CZ5 ceramics. The peak indexing for a tetragonal phase was adopted. (b) The enlarged (002) peaks. The inset figures in (a) illustrate the structures of CZ0 and CZ5 ceramics in an exaggerated manner.

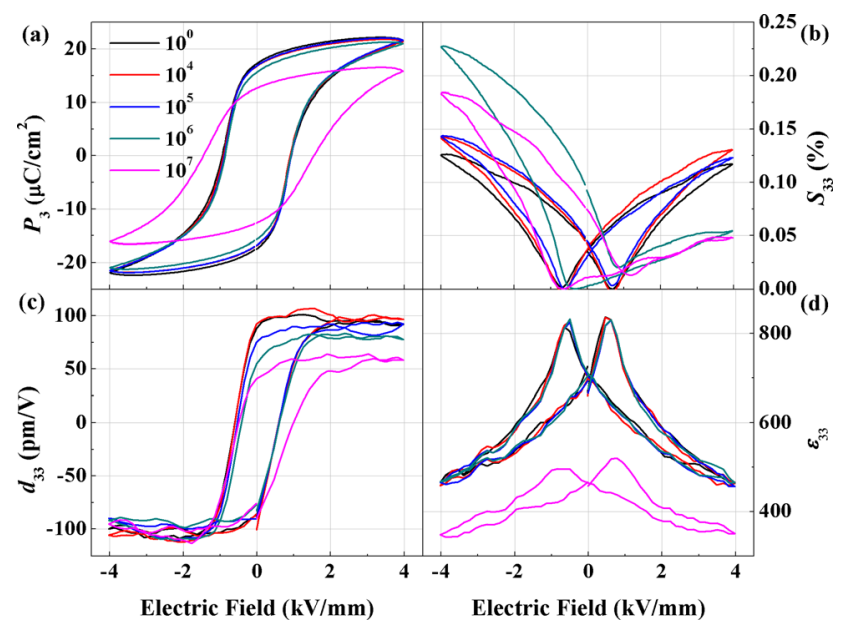

FIG. 2. (a) $P_{3}-E_{3}$, (b) $S_{33}-E_{3}$, (c) $d_{33}-E_{3}$, and (d) $\varepsilon_{33}-E_{3}$ of CZ0 ceramics before and after $10^{4}, 10^{5}, 10^{6}$, and $10^{7}$ bipolar fatigue cycles.

ceramics is less than $1^{\circ}$ different from $90^{\circ}$, so it is feasible to use "c/a ratio," a term normally applied in tetragonal ferroelectrics, for KNN with orthorhombic symmetry. The c/a ratios of CZ0 and CZ5 ceramics were 1.010 and 1.004, respectively, as calculated from the ratio of the sine values of peak locations for $(200) /(002)$. It is believed that the phase constitutions of these KNN-based ceramics are closely related to the fatigue resistance, as discussed later.

The evolution of both large signal (polarization $P_{3}$ and strain $S_{33}$ ) hysteresis and small signal (piezoelectric coefficient $d_{33}$ and permittivity $\varepsilon_{33}$ ) curves for CZ0 and CZ5 ceramics with bipolar electric cycling are displayed in Figs. 2 and 3, respectively. It is noticed that the strain output, piezoresponse, and permittivity of virgin CZ5 surpass those of $\mathrm{CZ0}$, which are possibly attributed to advantageous contribution of PPT effect in CZ5 over the deviated PPT in CZ0. ${ }^{26}$ Severe degradation of switchable polarization, piezoelectric response, and permittivity, as well as an asymmetry development in the $S_{33}-E_{3}$ curves were observed for the CZ0 ceramics as shown in Fig. 2. It is therefore reasonable to conclude that CZ0 ceramics are highly susceptible to bipolar cycling by the time of the $10^{7}$ cycles. However, there is also no discernable degradation of various properties for CZ5 when cycles were within $10^{6}$. In other words, the CZ5 ceramics are insensitive to electric cycling during the initial cycling

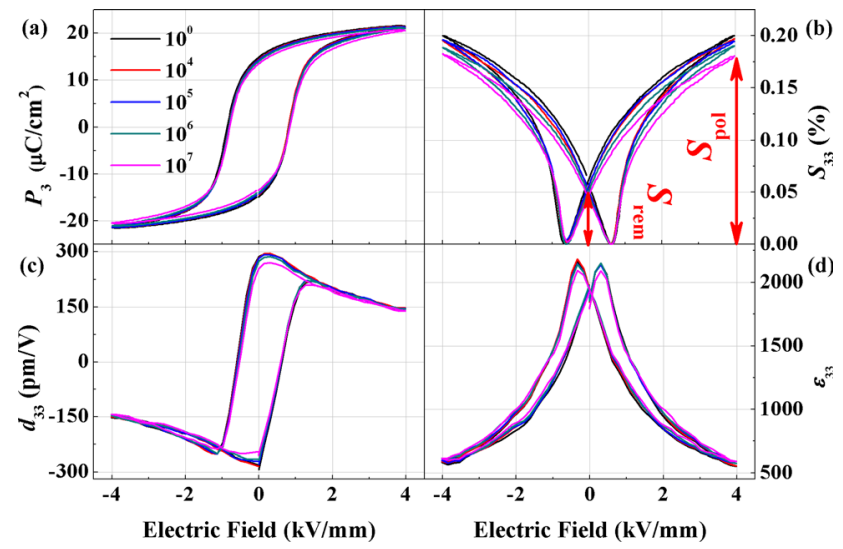

FIG. 3. (a) $P_{3}-E_{3}$, (b) $S_{33}-E_{3}$, (c) $d_{33}-E_{3}$, and (d) $\varepsilon_{33}-E_{3}$ of CZ5 ceramics before and after $10^{4}, 10^{5}, 10^{6}$, and $10^{7}$ bipolar fatigue cycles. 
stages. CZ5 began to degrade perceptibly after being cycled about $10^{7}$ times, as suggested by the slight decrease of switchable polarization $2 P_{\mathrm{r}}$, poling strain $S_{\text {pol }}$, piezoelectric coefficient $d_{33}$, and permittivity $\varepsilon_{33}$. It should be noted that the symmetry of $S_{33}-E_{3}$ curves of CZ5 remains intact and the positive applied field value for $S_{\text {pol }}$ deteriorated much less for CZ5 (10.1\% decrease) compared to the CZ0 samples (58.9\% decrease).

To quantify the degree of fatigue, the variation of the normalized $2 P_{\mathrm{r}}$ as a function of applied cycle number is presented in Fig. 4(a). The data of $0.94\left(\mathrm{Bi}_{0.5} \mathrm{Na}_{0.5}\right) \mathrm{TiO}_{3}-0.06 \mathrm{BaTiO}_{3}$ (BNT-BT), ${ }^{13} \quad 0.05 \mathrm{Bi}\left(\mathrm{Zn}_{0.5} \mathrm{Ti}_{0.5}\right) \mathrm{O}_{3}-0.40\left(\mathrm{Bi}_{0.5} \mathrm{~K}_{0.5}\right) \mathrm{TiO}_{3}-0.55$ $\left(\mathrm{Bi}_{0.5} \mathrm{Na}_{0.5}\right) \mathrm{TiO}_{3}(\mathrm{BNKZT}),{ }^{14}$ and PIC151 (a commercial soft PZT with the composition $\mathrm{Pb}_{0.99}\left[\mathrm{Zr}_{0.45} \mathrm{Ti}_{0.47}\left(\mathrm{Ni}_{0.33} \mathrm{Sb}_{0.67}\right)_{0.08}\right]_{3}$, PI Ceramics, Lederhose, Germany) ${ }^{18}$ from previous studies were also provided for comparison. The $2 P_{\mathrm{r}}$ of BNT-BT decreased dramatically within the first $10^{4}$ bipolar cycles and plateaued at a static value during the following cycles. It was suggested that a combination of a phase transition and of microstructure damage resulting from mechanical stress during the fatigue process may account for this fatigue behavior. ${ }^{13}$ It was demonstrated later that the fatigue-resistant phase can be stabilized by $\mathrm{CuO}$ addition, leading to significantly improved fatigue resistance of BNT-BT system. ${ }^{28}$

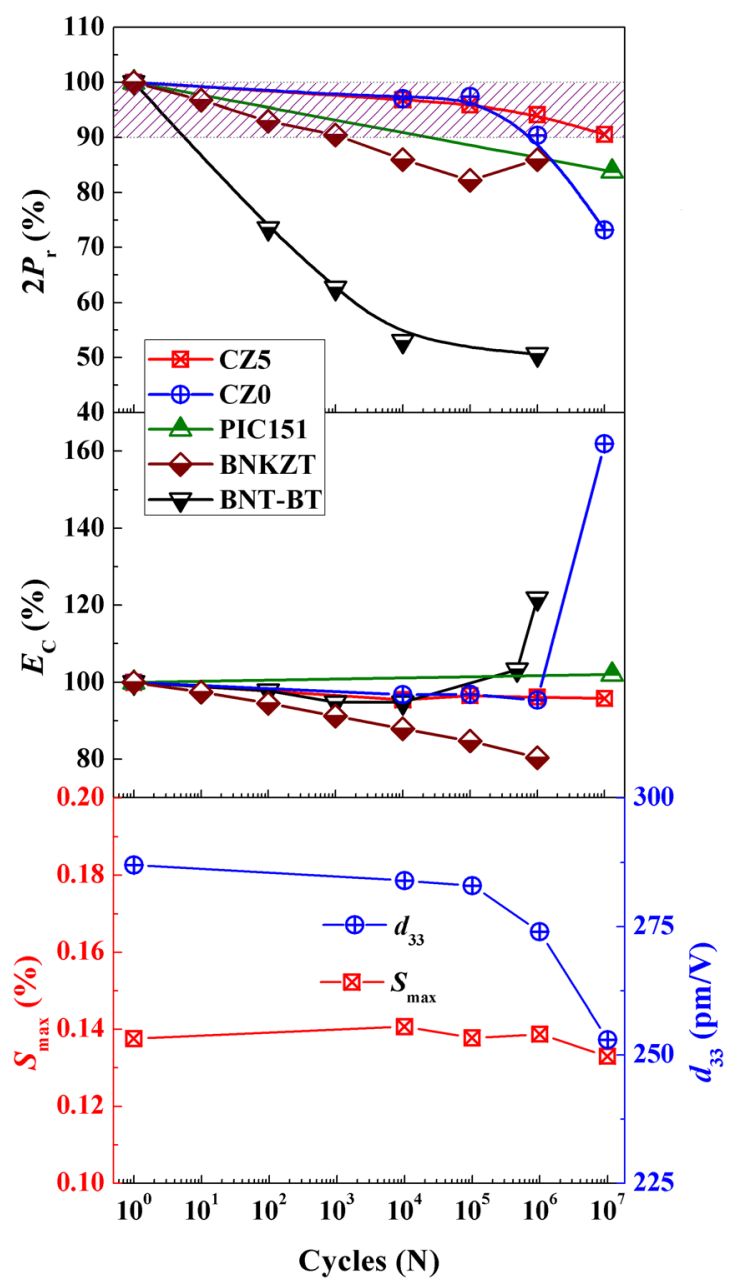

FIG. 4. The normalized (a) switchable polarization $2 P_{\mathrm{r}}$ and (b) coercive field $E_{\mathrm{C}}$ of $\mathrm{CZO}$ and $\mathrm{CZ} 5$ ceramics as functions of bipolar fatigue cycle number, and the corresponding values of BNT-BT, BNKZT, and PIC151 were plotted for comparison; (c) bipolar fatigue cycles dependence of maximum strain $S_{\max }$ as well as piezoelectric coefficient $d_{33}$ of CZ5 ceramics.
Additionally, promising fatigue resistance is achievable in the bismuth-based relaxors, e.g., BNKZT, through lowering the concentration of intrinsic defects. ${ }^{14}$ For PIC151 ceramics, a mild fatigue behavior was observed, possibly caused by the pinned domain walls due to agglomeration of charged defects in the bulk sample. ${ }^{18,29}$ In the case of CZO samples, an incubation time existed before serious fatigue behavior, analogous to a previous report about PZT. ${ }^{10}$ Most intriguingly, CZ5 presents highly enhanced fatigue resistance compared to the aforementioned compositions. The value of $2 P_{\mathrm{r}}$ for CZ5 decreased slightly with increasing cycle number and only $10 \%$ loss occurred when cycles reached up to $10^{7}$ times, while nearly $50 \%$ loss of $2 P_{\mathrm{r}}$ occurred for BNT-BT after $10^{6}$ cycles and about $30 \%$ loss for CZ0 after $10^{7}$ cycles. The possible origin of the high fatigue resistance for CZ5 may lie in its inherent mixed phase structure with coexisting orthorhombic and tetragonal and corresponding low c/a ratio (refer to the XRD pattern in Fig. 1). ${ }^{12}$

It is well known that the reversal of ferroelectric $\left(180^{\circ}\right)$ domains does not change the surrounding stress conditions; while ferroelastic (non-180 ${ }^{\circ}$ ) domain reorientation induces a mechanical stress field near the grain boundary and inside grains, since it demands coordinate accommodation by adjacent domains. ${ }^{30,31}$ The strain of the unfatigued CZ5 composition is greater than that of the CZ0 at the same field levels. Given a non-textured, polycrystalline arrangement of grains in both instances, the increased strain of the CZ5 could be expected to result in more mechanical stress fields within the sample. However, the improved fatigue resistance implies the opposite to be true. In CZ5, the composition effectively lowers the PPT temperature allowing for an enhanced mixture of phases to be present at room temperature. In previous work on PZT, it was shown that during mechanical loading, the ferroelastic coercive stress was decreased near the MPB, which coincided with lowered lattice distortions. ${ }^{32}$ In this region of partial phase coexistence, the critical energy barrier to switch domains was lowered. The $\mathrm{CZO}$ ceramics likewise had higher lattice distortion (c/a ratio), corresponding to a more dominant single orthorhombic phase of KNN with an increased energy barrier for ferroelastic domain switching. This resulted in a more severe fatigue process than in CZ5. For CZ5, the mixture of orthorhombic and tetragonal phases suggests that phase transitions and domain switching are facilitated and hence an expected lower coercive stress leads to accommodating domain switching rather than result in cracking. Consequently, CZ5 ceramics exhibit no trace of microstructural deformation, as depicted in Fig. 5(b), as compared to the stress induced microstructural damage below the electrodes of CZ0 shown in Fig. 5(a).

Fig. 4(b) depicts the bipolar cycle dependent $E_{\mathrm{C}}$ of these compositions. The variation of $E_{\mathrm{C}}$ for $\mathrm{CZ5}, \mathrm{PIC} 151$, and BNKZT differs from that of CZO and BNT-BT ceramics, where $E_{\mathrm{C}}$ increased dramatically at the end of fatigue process. Mechanisms related to field screening and/or microstructure damage, which may be responsible for significantly increased $E_{\mathrm{C}}$ after fatigue in CZ0 and BNT-BT, can therefore be excluded as potential causes of fatigue for $\mathrm{CZ5}$. $^{13,20}$ The recovery of the mild fatigue behavior of CZ5 also rejects the mechanisms of field screening and/or microstructure damage, since the deteriorated properties induced by the two 

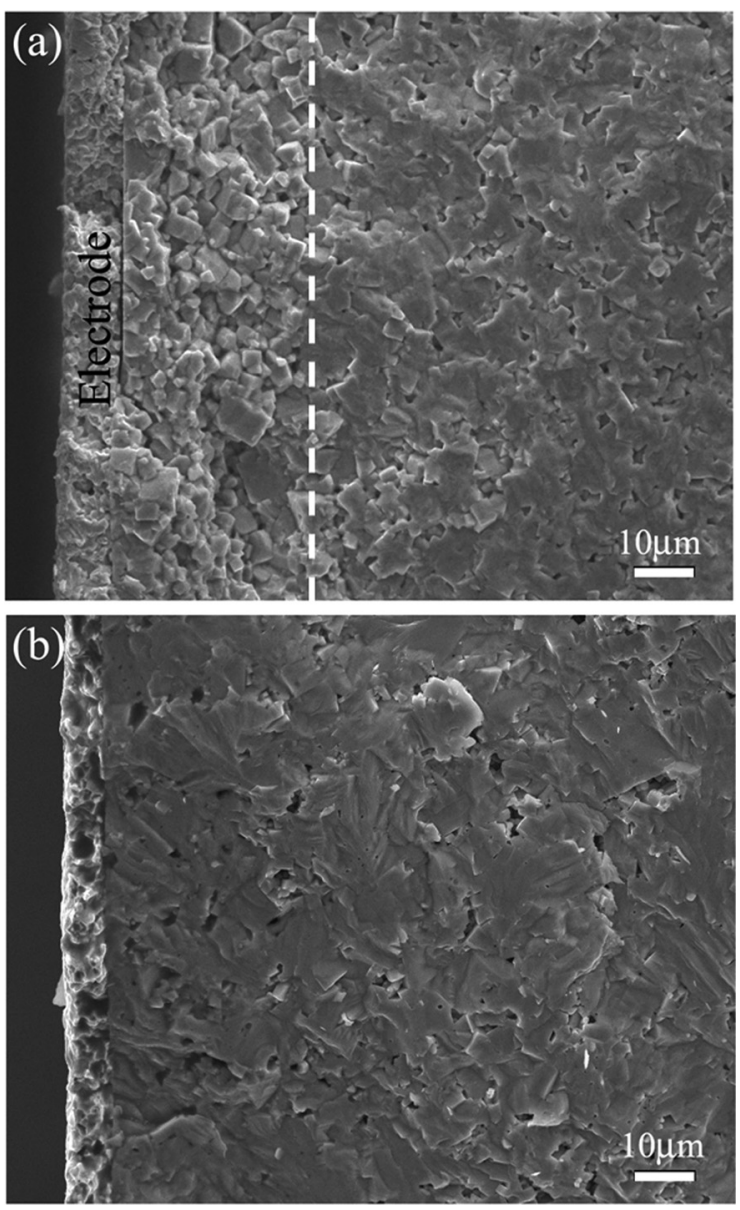

FIG. 5. SEM images of fracture surfaces of (a) CZ0 and (b) CZ5 ceramics after $10^{7}$ bipolar fatigue cycles.

mechanisms cannot be fully repaired by a simple thermal annealing treatment (refer to Fig. 6). ${ }^{33}$

The data for maximum strain $\left(S_{\max }\right.$, the difference between poling strain $S_{\text {pol }}$ and remnant strain $S_{\text {rem }}$ in bipolar strain curve, as illustrated in Fig. 3(b)) and the piezoelectric coefficient $d_{33}$ of CZ5 ceramics are given in Fig. 4(c). The $d_{33}$ reveals negligible loss in the first $10^{5}$ cycles, and then the decrease of $d_{33}$ becomes noticeable in the following cycles, exhibiting a similar trend as the $2 P_{\mathrm{r}}$. The gentle deterioration of $d_{33}$ may be ascribed to lowered domain wall mobility, possibly due to defect-induced domain wall pinning. This is proposed as the cause of the minor fatigue process observed for CZ5 ceramics, as no evidence of mechanical deformation emerged. Compared to $2 P_{\mathrm{r}}$ and $d_{33}$ of CZ5, the bipolar fatigue process has a negligible impact on $S_{\max }$, which is an important feature for application of actuators. ${ }^{26,34}$ Thus, $S_{\max }$ barely varies with increasing fatigue cycles, suggesting that the domains are still reversible under the stimulation of large electric fields even when the domain walls are partially pinned.

For bipolar ${ }^{35}$ or unipolar ${ }^{36}$ fatigued PZT ceramics, it was reported that the properties can be at least partially recovered through a thermal annealing treatment. In the case of bipolar cycling, temperatures well-above the Curie temperature $T_{\mathrm{C}}$ were required to remove the fatigue effect, ${ }^{35}$ but unipolar fatigue effects could be completely annealed away at significantly lower temperatures (around $\left.T_{\mathrm{C}}\right){ }^{36}$ The

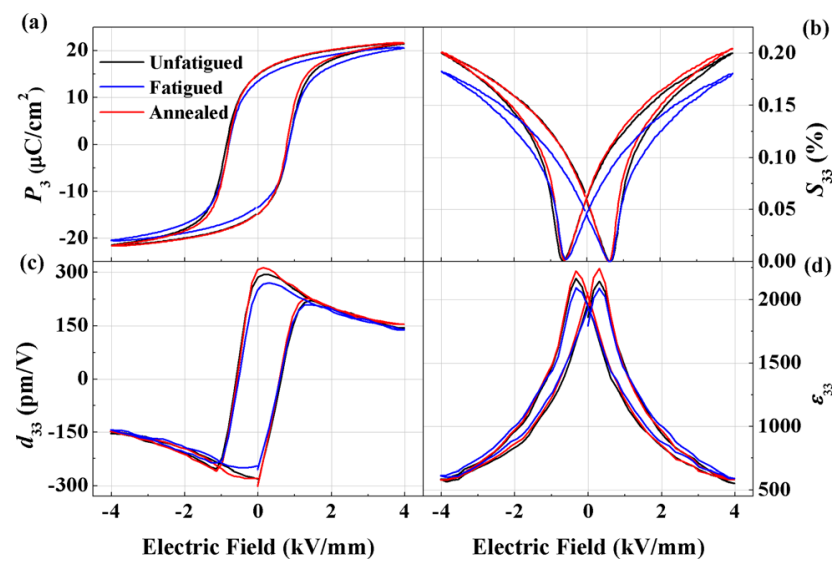

FIG. 6. Effect of thermal annealing on (a) $P_{3}-E_{3}$, (b) $S_{33}-E_{3}$, (c) $d_{33}-E_{3}$, and (d) $\varepsilon_{33}-E_{3}$ of $\mathrm{CZ} 5$ ceramics comparing the unfatigued, fatigued $\left(10^{7}\right.$ cycles), and annealed $\left(500^{\circ} \mathrm{C}\right.$ for $\left.4 \mathrm{~h}\right)$ states.

stability of the fatigue characteristics of the CZ0 and CZ5 samples were also tested against thermal treatment after fatigue. Fig. 6 provides the bipolar large signal as well as small signal curves of unfatigued, fatigued $\left(10^{7}\right.$ cycles $)$, and annealed $\left(500{ }^{\circ} \mathrm{C}\right.$ for $\left.4 \mathrm{~h}\right)$ states of $\mathrm{CZ} 5$ ceramics. It should be mentioned that the annealing temperature is much higher than $T_{\mathrm{C}}\left(192{ }^{\circ} \mathrm{C}\right.$ for $\left.\mathrm{CZ5}\right)$. It can be observed that the thermal annealing treatment can lead to a full recovery of the fatigued properties of CZ5, agreeing well with the study of PZT with domain pinning described as the fatigue mechanism. ${ }^{35}$ However, recovery did not occur for CZ0 ceramics owing to the mechanical deterioration (curves not shown).

In summary, the bipolar fatigue behaviors of KNNbased lead-free piezoceramics were investigated systematically. A remarkably improved bipolar fatigue resistance was achieved in $\mathrm{CaZrO}_{3}$-modified $\mathrm{KNN}$ ceramics benefiting from the reduced lattice distortion and an increased mixture of orthorhombic and tetragonal phases. Combining the previously reported temperature-cycling-insensitive strain behaviors $^{26}$ and unipolar-cycling-insensitive strain behaviors, ${ }^{17}$ the exceptionally good bipolar fatigue resistance favors the successful implementation of $\mathrm{CaZrO}_{3}$-modified $\mathrm{KNN}$ ceramics for certain applications.

This work was supported by National Nature Science Foundation of China (Grant Nos. 51332002, 51302144, 51221291, and 51211140345), Specialized Research Fund for the Doctoral Program of Higher Education (Grant No. 20130002120031), and Tsinghua University Initiative Scientific Research Program (Grant No. 20131089230). K. Wang acknowledges the Alexander von Humboldt foundation for a fellowship.

${ }^{1}$ Y. Saito, H. Takao, T. Tani, T. Nonoyama, K. Takatori, T. Homma, T. Nagaya, and M. Nakamura, Nature 432(4), 84 (2004).

${ }^{2}$ J. Rödel, W. Jo, K. T. P. Seifert, E.-M. Anton, T. Granzow, and D. Damjanovic, J. Am. Ceram. Soc. 92(6), 1153 (2009).

${ }^{3}$ T. R. Shrout and S. J. Zhang, J. Electroceram. 19(1), 113 (2007).

${ }^{4}$ J.-F. Li, K. Wang, F.-Y. Zhu, L.-Q. Cheng, and F.-Z. Yao, J. Am. Ceram. Soc. 96(12), 3677 (2013).

${ }^{5}$ E. Hollenstein, M. Davis, D. Damjanovic, and N. Setter, Appl. Phys. Lett. 87(18), 182905 (2005).

${ }^{6}$ R. Zuo and J. Fu, J. Am. Ceram. Soc. 94(5), 1467 (2011). 
${ }^{7}$ J. Zushi, T. Ariizumi, S. Kojima, R. Wang, and H. Bando, Jpn. J. Appl. Phys., Part 1 52, 07HB02 (2013).

${ }^{8}$ J. Yao, J. Li, D. Viehland, Y. Chang, and G. L. Messing, Appl. Phys. Lett. 100(13), 132902 (2012).

${ }^{9}$ K. Wang and J.-F. Li, Adv. Funct. Mater. 20(12), 1924 (2010).

${ }^{10}$ J. Nuffer, D. C. Lupascu, and J. Rödel, Acta Mater. 48, 3783 (2000).

${ }^{11}$ H. Wang, T. Matsunaga, H.-T. Lin, and A. M. Mottern, Smart Mater. Struct. 21(2), 025009 (2012).

${ }^{12}$ D. A. Hall, T. Mori, T. P. Comyn, E. Ringgaard, and J. P. Wright, J. Appl. Phys. 114(2), 024103 (2013).

${ }^{13}$ Z. Luo, J. Glaum, T. Granzow, W. Jo, R. Dittmer, M. Hoffman, and J. Rödel, J. Am. Ceram. Soc. 94(2), 529 (2011).

${ }^{14}$ E. A. Patterson and D. P. Cann, Appl. Phys. Lett. 101(4), 042905 (2012).

${ }^{15}$ H. Simons, J. Glaum, J. E. Daniels, A. J. Studer, A. Liess, J. Rödel, and M. Hoffman, J. Appl. Phys. 112(4), 044101 (2012).

${ }^{16}$ E. A. Patterson and D. P. Cann, IEEE Trans. Ultrason. Ferroelectr. Freq. Control 58(9), 1835 (2011).

${ }^{17}$ F.-Z. Yao, J. Glaum, K. Wang, W. Jo, J. Rödel, and J.-F. Li, Appl. Phys. Lett. 103(19), 192907 (2013).

${ }^{18}$ J. Glaum, T. Granzow, and J. Rödel, J. Appl. Phys. 107(10), 104119 (2010).

${ }^{19}$ S. M. Yang, T. H. Kim, J.-G. Yoon, and T. W. Noh, Adv. Funct. Mater. 22(11), 2310 (2012).

${ }^{20}$ N. Balke, H. Kungl, T. Granzow, D. C. Lupascu, M. J. Hoffmann, and J. Rödel, J. Am. Ceram. Soc. 90(12), 3869 (2007).

${ }^{21}$ J. Nuffer, D. C. Lupascu, and J. Rödel, J. Eur. Ceram. Soc. 21, 1421 (2001).
${ }^{22}$ Q. Y. Jiang, E. C. Subbarao, and L. E. Cross, J. Appl. Phys. 75(11), 7433 (1994).

${ }^{23}$ S. Zhang, R. Xia, H. Hao, H. Liu, and T. R. Shrout, Appl. Phys. Lett. 92, 152904 (2008).

${ }^{24}$ Y. Dai, X. Zhang, and G. Zhou, Appl. Phys. Lett. 90(26), 262903 (2007).

${ }^{25}$ X. Wang, J. Wu, D. Xiao, J. Zhu, X. Cheng, T. Zheng, B. Zhang, and X. Lou, J. Am. Chem. Soc. 136(7), 2905 (2014).

${ }^{26}$ K. Wang, F.-Z. Yao, W. Jo, D. Gobeljic, V. V. Shvartsman, D. C. Lupascu, J.-F. Li, and J. Rödel, Adv. Funct. Mater. 23(33), 4079 (2013).

${ }^{27}$ K. Wang and J.-F. Li, Appl. Phys. Lett. 91(26), 262902 (2007).

${ }^{28}$ M. Ehmke, J. Glaum, W. Jo, T. Granzow, and J. Rödel, J. Am. Ceram. Soc. 94(8), 2473 (2011).

${ }^{29}$ J. Glaum, T. Granzow, L. A. Schmitt, H.-J. Kleebe, and J. Rödel, Acta Mater. 59(15), 6083 (2011).

${ }^{30}$ B. Li, J. E. Blendell, and K. J. Bowman, J. Am. Ceram. Soc. 94(10), 3192 (2011).

${ }^{31}$ F. Rubio-Marcos, J. J. Romero, D. A. Ochoa, J. E. García, R. Perez, and J. F. Fernandez, J. Am. Ceram. Soc. 93(2), 318 (2010).

${ }^{32}$ Y.-H. Seo, D. Franzbach, J. Koruza, A. Benčan, B. Malič, M. Kosec, J. Jones, and K. Webber, Phys. Rev. B 87(9), 094116 (2013).

${ }^{33}$ Q. Jiang, W. Cao, and L. E. Cross, J. Am. Ceram. Soc. 77(1), 211 (1994).

${ }^{34}$ W. Jo, R. Dittmer, M. Acosta, J. Zang, C. Groh, E. Sapper, K. Wang, and J. Rödel, J. Electroceram. 29(1), 71 (2012).

${ }^{35}$ J. Nuffer, D. C. Lupascu, and J. Rödel, Appl. Phys. Lett. 80(6), 1049 (2002).

${ }^{36}$ C. Verdier, D. C. Lupascu, and J. Rödel, Appl. Phys. Lett. 81(14), 2596 (2002). 